\title{
Les premiers chrétiens dans le territoire de la France actuelle
}

Alain Valais

\section{OpenEdition}

12 Journals

Édition électronique

URL : http://journals.openedition.org/rao/1431

DOI : 10.4000/rao.1431

ISSN : 1775-3732

Éditeur

Presses universitaires de Rennes

\section{Édition imprimée}

Date de publication : 31 décembre 2010

Pagination : 231-234

ISBN : 978-2-7535-1383-9

ISSN : 0767-709X

\section{Référence électronique}

Alain Valais, "Les premiers chrétiens dans le territoire de la France actuelle », Revue archéologique de I'Ouest [En ligne], 27 | 2010, mis en ligne le 25 février 2012, consulté le 03 décembre 2020. URL : http:// journals.openedition.org/rao/1431; DOI : https://doi.org/10.4000/rao.1431

Ce document a été généré automatiquement le 3 décembre 2020.

Tous droits réservés 


\title{
Les premiers chrétiens dans le territoire de la France actuelle
}

\author{
Alain Valais
}

\section{RÉFÉRENCE}

Paris Poulain, D., Istria, D. et Nardi Combescure, S. (dir.), 2009 - Les premiers chrétiens dans le territoire de la France actuelle. Hagiographie, épigraphie et archéologie : nouvelles approches et perspectives de recherche (Actes du colloque international d'Amiens, Université de Picardie Jules-Verne, Faculté des Arts, 18 -20 janvier 2007), Rennes, PUR, coll. « Archéologie et Culture », 252 p. (ISBN 278-2-7535-0854-5; $24 €$ )

1 Ce volume constitue la publication des actes d'un colloque international qui s'est déroulé à Amiens du 18 au 20 janvier 2007. Le sommaire de cet ouvrage de 250 pages s'articule autour de trois thèmes: les premiers temps chrétiens, apport des sources historiques, hagiographiques et épigraphiques ; la topographie des cités chrétiennes et des agglomérations secondaires à la lumière de découvertes archéologiques récentes et enfin le christianisme en milieu rural. La plupart des contributions présente les résultats de recherches récentes, essentiellement issues de fouilles préventives et qui concernent le paysage monumental, de l'Antiquité tardive à la fin de la période mérovingienne.

2 Le premier thème concerne l'étude de la christianisation du nord de la Gaule mérovingienne au travers de récits de vies de saints (par B. Meijns et C. Mériaux), de l'identification des lieux où ils ont été martyrisés et souvent enterrés. Ces sites, la plupart du temps implantés sur des voies antiques, sont souvent à l'origine de basiliques rurales qu'il est parfois possible d'identifier. Cette contribution est suivie d'un texte de L. Pietri à propos d'un ascète stylite, Vulfilaicus, qui a contribué à sa manière à l'évangélisation des Ardennes. Dans cette partie de l'ouvrage, il est également question de la royauté franque et de la christianisation aux temps de Childebert I ${ }^{\text {er }}$, où $\mathrm{B}$. Dumézil montre que, bien plus que sous Clovis son père, sont 
encouragées les conversions durant un règne déjà assez largement imprégné de principes chrétiens. Les pratiques païennes apparaissent alors dans les sources comme plus fermement combattues.

3 Deux contributions présentent ensuite des travaux épigraphiques, l'une sur des coupes en verre à décors chrétiens découvertes en Picardie et une autre issue de l'étude de mobiliers archéologiques figurant le même type de motif découverts en Belgique et dans le nord de la Gaule. Après avoir présenté leur corpus, les auteurs (respectivement S. Nardi Combescure et $\mathrm{S}$. Lambot) se demandent dans quelle mesure ces signes chrétiens témoignent du sentiment religieux de leurs propriétaires où s'ils ne constituent que des motifs ornementaux... Une relecture de la Vie de Saint Didier évêque de Cahors, par F. Prévot, autour du thème de la construction d'une ville épiscopale au $\mathrm{VII}^{\mathrm{e}}$ siècle, vient clore ce chapitre...

Dans la seconde partie de l'ouvrage, les contributions sont issues de découvertes archéologiques réalisées principalement en contexte urbain. M. Heijmans présente une église paléochrétienne qu'il a exhumée dans l'enclos Saint-Césaire à Arles. Cet édifice, fouillé au cours de plusieurs opérations menées entre 2003 et 2006, se compose d'un choeur de $20 \mathrm{~m}$ d'ouverture, polygonal à l'extérieur et semi-circulaire à l'intérieur. Une abside y est incluse sans que l'on sache s'il s'agit d'un second chevet appartenant à une autre phase de construction ou d'un seul bâtiment doté d'un banc presbytéral. Le mur semi-circulaire le plus vaste s'appuie sur un mur qui correspond soit à un transept soit au début d'une nef. Ces constructions, établies au sein d'un vaste édifice public du Haut-Empire, s'étaleraient du milieu du $\mathrm{IV}^{\mathrm{e}}$ siècle à la première moitié du $\mathrm{v}^{\mathrm{e}}$, l'ensemble paraissant abandonné dès la fin $\mathrm{du}_{\mathrm{VI}}^{\mathrm{e}}$ ou au début du siècle suivant...

5 La contribution suivante, rédigée par $M$. Moliner, dresse un panorama de ce que Marseille a livré sur ces premiers siècles de chrétienté. Un état de lieu est dressé, avec notamment les découvertes réalisées au xix ${ }^{\mathrm{e}}$ siècle autour de l'abbaye Saint-Victor, un baptistère de la fin du IV siècle découvert lors de la reconstruction de la cathédrale et différentes nécropoles antiques et paléochrétiennes. Ces quelques données anciennes ont été totalement renouvelées par les différentes fouilles de sauvetage qui ont ponctué les grands chantiers de rénovation de la ville depuis les années quatre-vingt. Outre un édifice à plan centré mis en évidence à Saint-Victor, édifice funéraire établi sur le lieu présumé de la tombe de ce martyr du $\mathrm{III}^{\mathrm{e}}$ siècle, notons également la présentation d'une église funéraire inédite découverte rue Malaval. Dégagé au deux tiers de sa surface, ce sanctuaire est doté d'une abside semi-circulaire de 6,10 m d'ouverture qui abritait une sépulture double particulièrement vénérée. Ce sanctuaire est actif durant le $\mathrm{v}^{\mathrm{e}}$ siècle...

6 La ville de Valence constitue également un chapitre intéressant de cet ouvrage avec la présentation des résultats des fouilles de 2003-2004 réalisées sur la place des Ormeaux par F. Gabayet. Dans un secteur de la ville antique occupé au premier siècle de notre ère, un quartier résidentiel est édifié dans le courant $d u v^{e}$ siècle au sud de la cathédrale primitive et de son baptistère. Ces bâtiments, établis autour de deux cours, sont associés à une petite église et à un ensemble thermal. Ce complexe est interprété comme faisant partie du quartier résidentiel de l'évêque. Le groupe épiscopal primitif, qui pourrait être construit dès le $\mathrm{IV}^{\mathrm{e}}$ siècle, n'est clairement identifié qu'au siècle suivant quand un premier bâtiment à abside est construit. L'ensemble évolue ensuite tout au long du haut Moyen Âge, notamment avec la construction de plusieurs églises 
qui se succèdent. La dernière, qui remonte aux $\mathrm{x}^{\mathrm{e}} / \mathrm{xII}^{\mathrm{e}}$ siècles, semble associée au cimetière paroissial des Ormeaux qui est utilisé jusqu'au XviI ${ }^{\mathrm{e}}$ siècle.

7 Ce chapitre s'achève avec une contribution de J. Guyon qui dresse une synthèse sur la topographie chrétienne des cités du Midi de la France. Il constate que les données disponibles sont souvent fragmentaires; il précise en particulier que sur les 74 villes épiscopales du Midi, l'archéologie et les sources n'ont rien livré sur le sujet pour un quart d'entre-elles. L'auteur insiste sur l'imprécision dans la chronologie de ces édifices dont la plupart remonte au ve siècle au sens large. Le groupe épiscopal constitué d'une cathédrale, d'un baptistère, de locaux de service et de la résidence de l'évêque constitue alors le principal centre chrétien de l'espace urbain. Il est construit au centre de la ville ou parfois sur un point dominant comme une colline à Arles ou en front de mer à Marseille. Si les autres édifices chrétiens établis dans les villes sont rares, il en est tout autrement des établissements suburbains qui représentent les deux tiers des sanctuaires. Il s'agit le plus souvent de basiliques funéraires édifiées sur la tombe d'un saint ou sur des reliques de martyrs autour desquelles on désire se faire inhumer. Ce phénomène conduit au développement de cimetières et de faubourgs qui tiendront un rôle majeur dans l'évolution des villes médiévales et modernes. Beaucoup de ces établissements suburbains deviendront alors le siège d'abbayes qui rivaliseront souvent avec les groupes épiscopaux... L'auteur insiste enfin sur les agglomérations secondaires qui se dotent également de sanctuaires à la manière de ces cités épiscopales. De la même façon, en contexte rural, on voit apparaître dès le v viècle des mausolées qui sont rapidement transformés en édifices de culte parfois associés à un baptistère.

8 Les quelques contributions qui suivent évoquent le site de Saint-Quentin, le groupe épiscopal d'Ajaccio, le baptistère de Limoges, la basilique des Champs à Rezé (44) et le baptistère de Roanne. C. Sapin a repris l'étude de la collégiale de Saint-Quentin bâtie autour de la tombe de ce saint, à la lumière des textes, de plusieurs campagnes de sondages anciens (xIX siècle, 1926 et 1955) et surtout de ses investigations archéologiques de 2004 et 2007. L'histoire de cet édifice commence dès l'antiquité tardive (phase 2) avec une série d'aménagements autour de la tombe du saint. Un sanctuaire est construit au $\mathrm{VI}^{\mathrm{e}}$ siècle (phase 3) et lui sont associées d'autres sépultures privilégiées. Plus tard (phase $4, \mathrm{VII}^{\mathrm{e}}$ siècle), un chevet plat et une nef sont édifiés au moment où, selon les textes, la tombe vénérée est déplacée derrière l'autel de ce nouvel édifice. L'état carolingien (phase 5) voit la construction d'une abside et d'une crypte dont le plan sera totalement modifié au XIII ${ }^{\mathrm{e}}$ siècle.

9 D. Istria présente ensuite la fouille préventive qui a conduit à la découverte du groupe épiscopal d'Ajaccio. Ces vestiges ont été exhumés à une dizaine de mètres au sud de la cathédrale détruite au XVIII ${ }^{e}$ siècle. La zone est occupée dès le Ier siècle par un habitat qu'il reste à localiser et par une zone de sépultures de la même période, connue depuis le $\mathrm{XIX}^{\mathrm{e}}$ siècle. Les vestiges découverts en 2005 sont constitués d'une cuve baptismale établie dans une abside. Cet ensemble, que l'auteur attribue aux premières décennies $\mathrm{du} \mathrm{VI}^{\mathrm{e}}$ siècle, appartient à un bâtiment plus vaste et plus ancien dont il ne subsiste qu'un seul mur. Plus tard, ce baptistère est partiellement détruit ; la nouvelle cuve qui est aménagée à proximité appartient dès lors à un nouvel édifice construit entre la seconde moitié $\mathrm{du} \mathrm{VI}^{\mathrm{e}}$ siècle et le début du siècle suivant. Cette église, associée à des sépultures regroupées autour de son abside, est détruite vers 1100. 
10 Un article de J. Denis concerne ensuite la datation du baptistère de Limoges. On sait qu'une modeste église paroissiale dédiée à Saint-Jean-Baptiste existait jusqu'à la Révolution devant le portail nord de la cathédrale. Des sondages réalisés à cet endroit en 2004 ont débouché sur la découverte d'un baptistère paléochrétien et sa fouille en 2005. L'édifice, de plan hexagonal, est organisé autour d'une cuve dont deux états ont pu être mis en évidence. Le bâtiment connaît également de nombreuses modifications, notamment l'ajout d'une abside et d'un autel avant sa démolition au XIII siècle et son remplacement pas cette église Saint-Jean Baptiste consacrée en 1219. La datation de ce baptistère restait aléatoire en raison de la pauvreté du mobilier issu de la fouille. On savait juste que la construction était établie sur des niveaux du $\mathrm{IV}^{\mathrm{e}}$ siècle et que les textes la faisaient remonter avant 475 . Des datations $14 \mathrm{C}$ sont venues confirmer ces informations en indiquant une période de construction comprise entre 320 et 420 pour le baptistère et entre 430 et 550 pour son abside.

11 La contribution suivante, rédigée par L. Pirault, présente la basilique des Champs-SaintMartin découverte en 2001 à Rezé (Loire-Atlantique), une agglomération antique et surtout un port établi sur la rive sud de la Loire un peu en aval de Nantes. Cet édifice de $750 \mathrm{~m}^{2}$ se compose d'un chevet plat et d'une nef longue de $19,60 \mathrm{~m}$ pour $14 \mathrm{~m}$ de largeur, divisée longitudinalement par un mur. Lors d'une première phase, un espace privilégié constitué par une plate forme quadrangulaire appuyée contre le mur de chevet est entourée par deux alignements de trois supports. L'interprétation religieuse de cet ensemble repose sur la présence d'un autel établi dans la partie oriental de l'édifice. L'auteur s'interroge néanmoins sur les aménagements et leur fonction liturgique à l'intérieur de ce bâtiment énigmatique singulier au début $\mathrm{du}_{\mathrm{VI}}{ }^{\mathrm{e}}$ siècle.

Le texte suivant, rédigé par $M$. Le Nezet-Célestin, concerne la découverte d'un baptistère à Roanne en 2005. Cette ville d'origine antique n'était pas mentionnée dans les textes avant l'an Mil et, en dehors de quelques sarcophages, les découvertes du haut Moyen Âge y demeuraient rares jusqu'à cette découverte. Cet édifice baptismal entouré d'un cimetière est implanté au sein d'un quartier antique abandonné depuis le $\mathrm{III}^{\mathrm{e}}$ siècle. Son plan se compose d'un espace rectangulaire où sont aménagés les deux états d'une cuve baptismale. Ce bâtiment dispose d'une abside orientale. Se référant aux exemples les plus proches que sont Lyon et Grenoble, l'auteur, qui dispose de peu d'élément de datation issus de la fouille, propose d'en attribuer la construction à la fin $\mathrm{du} \mathrm{v}^{\mathrm{e}}$ siècle ou au début $\mathrm{du}$ siècle suivant. Ajoutons que les sépultures les plus anciennes du cimetière remontent $a u v^{e}$ siècle et à la première moitié du $\mathrm{VI}^{\mathrm{e}}$.

13 Un texte collectif s'attache ensuite à la présentation d'églises rurales découvertes pour trois d'entre-elles au cours de fouilles préventives dans le nord de la France. Ces églises, parfois en bois, sont rares malgré le nombre de sites fouillés. De plus, les vestiges en sont souvent difficiles à identifier dans des zones où se concentrent les sépultures. Quatre sites en particulier, qui sont associés chaque fois à un habitat, font l'objet d'une présentation détaillée: Saleux (Aisne), Mondeville (Calvados) Tournedos-sur-Seine (Seine-Maritime) et Serris (Seine-et-Marne).

14 Saleux, fouillé en 1993 par I. Catteddu, constitue un remarquable exemple d'habitat rural occupé $d u \mathrm{VII}^{\mathrm{e}}$ au $\mathrm{XI}^{\mathrm{e}}$ siècle. Un cimetière et une église y ont été relevés. Entre le milieu du VII ${ }^{\mathrm{e}}$ siècle et une partie du siècle suivant, un premier édifice en bois abrite un sarcophage autour duquel s'organisent d'autres tombes. Un peu plus tard (milieu du $\mathrm{VIII}^{\mathrm{e}}$ siècle), un second bâtiment, constitué d'une nef et d'un chœur rectangulaire, vient 
remplacer ce premier état. Les sépultures sont dès lors organisées sur les orientations $\mathrm{du}$ nouvel édifice. Au cours des $\mathrm{IX}^{\mathrm{e}}$ et $\mathrm{x}^{\mathrm{e}}$ siècles, l'église est progressivement reconstruite en dur.

15 En Normandie, quelques découvertes d'églises du haut Moyen Âge ont également été mises en évidence, notamment à Mondeville sur le site de la Delles-Saint-Martin. L'édifice repéré au sein d'un habitat réutilise en partie un bâtiment antique. Cet ensemble est associé à des sépultures dont certaines, considérées comme contemporaines de l'église, remontent au vile siècle. À la fin du $\mathrm{IX}^{\mathrm{e}} /$ début du $\mathrm{X}^{\mathrm{e}}$ siècle, une seconde construction vient remanier la première en l'agrandissant.

16 Cette association entre habitat rural et église est également illustrée sur le site de Tournedos-sur-Seine, un site fouillé par F. Carré entre 1986 et 1994. Proche d'une zone d'habitat créée dans la seconde moitié du vII siècle, un cimetière principal et deux périphériques ont pu être également fouillés. Les tombes les plus anciennes, qui appartiennent à la zone d'inhumation principale, remontent au milieu du $\mathrm{VII}^{\mathrm{e}}$ siècle. Elles sont disposées autour d'un bâtiment rectangulaire excavé antérieur, dont la fonction ne semble pas domestique. Alors qu'il est encore en service, une église de près de $40 \mathrm{~m}^{2}$ constituée de deux alignements de poteaux est construite à proximité, au plus tôt au milieu du vil ${ }^{e}$ siècle et avant la seconde moitié du siècle suivant.

17 Le site de Serris, fouillé par F. Gentili de 1989 à 1997 sur une vingtaine d'hectares, a permis d'explorer un habitat occupé du $\mathrm{VII}^{\mathrm{e}}$ au $\mathrm{XI}^{\mathrm{e}}$ siècles, associé au nord à deux bâtiments relevés au centre d'un cimetière. Ces deux constructions rectangulaires utilisent la pierre, matériau réservé sur le site aux bâtiments privilégiés. Leur environnement livre également des fragments de verre à vitre. Le bâtiment le plus ancien, probablement aménagé autour d'une tombe, semble plutôt remplir une fonction funéraire alors que le second, entouré de sépultures des $\mathrm{VII}^{\mathrm{e}} / \mathrm{VIII}^{\mathrm{e}}$ siècles, semble constituer une église... Dans cet article également, sont évoquées quelques exemples d'églises rurales du haut Moyen Âge découvertes en Normandie.

18 Deux contributions, la première sur les « premiers temps chrétiens dans le sud-ouest de la France ", par C. Delaplace, et la seconde sur les «premiers temps chrétiens dans les campagnes de Novempopulanie ( $\mathrm{IV}^{\mathrm{e}}-\mathrm{X}^{\mathrm{e}}$ siècle) ", rédigée par M.-G. Colin, viennent clore cet ouvrage.

19 L'ensemble des données issues de ce volume extrêmement riche, qu'elles soient d'origine urbaine ou rurale, conduisent souvent à une relecture des sources écrites et à une réévaluation quand elles existent des découvertes anciennes.

20 D'une manière générale, l'accent est mis dans la plupart des contributions sur les difficultés rencontrées à dater des vestiges généralement mal conservés en raison des innombrables remaniements qu'ils ont subis tout au long de leur longue histoire, l'implantation de ces groupes épiscopaux, de ces divers sanctuaires urbains et ruraux ayant le plus souvent très peu varié depuis l'origine. En dehors des groupes épiscopaux, plusieurs communications montrent en outre que dès le début du haut Moyen Âge, autour des édifices funéraires hors les murs en particulier, on cherche déjà à se rapprocher de la tombe du saint ou des reliques des martyrs, phénomène de concentration des sépultures que l'on retrouve un peu plus tard en contexte rural...

21 Les sites présentés dans ce volume, riches en données inédites, constituent la preuve du dynamisme de l'Antiquité tardive, période qui voit dès les $\mathrm{IV}^{\mathrm{e}}$ et $\mathrm{v}^{\mathrm{e}}$ siècles au sein des capitales régionales de l'ancienne Gaule romaine, la construction de complexes 
monumentaux qui vont plus tard profondément marquer la topographie des villes médiévales. Ces phénomènes se reproduisent également dans les agglomérations secondaires qui se dotent, au moins dans le sud de la France, d'une église et d'une cuve baptismale à la manière du chef-lieu de cité. De la même façon, et c'est probablement un des principaux apports de ces vingt dernières années de recherche, le monde rural n'est pas en reste et des églises, dont la part est encore difficile à percevoir, sont construites durant les $\mathrm{VI}^{\mathrm{e}} / \mathrm{VII}^{\mathrm{e}}$ siècles. En revanche, malgré les nombreux habitats ruraux fouillés en préventif dans la moitié nord de la France, exceptionnels sont ceux qui disposent dès cette période d'une église et d'un cimetière. Il est d'autre part intéressant de souligner que, pour les quelques exemples connus, la plupart sont en bois, type d'architecture maintes fois évoqué dans les sources et encore très peu mis en évidence sur le terrain...

Cet ouvrage dense dresse d'abord un panorama extrêmement intéressant sur les débuts du christianisme en France, mais également un état de la recherche où se mêlent avec pertinence les apports des sources historiques, hagiographiques, épigraphiques et archéologiques.

\section{AUTEURS}

\section{ALAIN VALAIS}

(Inrap Pays de la Loire) 